\title{
Innovación y Política Tecnológica: el caso del Sector Transformador y el Sector Servicios a Empresas
}

\author{
Innovation and R\&D Policy: the Case of \\ Transforming Sector and Business Services Sector
}

\author{
María Soledad Castaño Martínez ${ }^{1}$ \\ Universidad de Castilla-La Mancha (España)
}

Recibido el 3 de octubre de 2011, aceptado el 18 de enero de 2012

$\mathrm{N}^{\mathrm{o}}$ de clasificación JEL: O31 O39, N60

DOI: $10.5295 / \mathrm{cdg} .110307 \mathrm{mc}$

\section{Resumen:}

Este trabajo analiza algunos de los determinantes de la innovación de producto en el caso de los emprendedores del sector transformador y del sector servicios a empresas. Entre estos factores se encuentran el capital humano, la tecnología el grado de competencia del mercado, las expectativas y el grado de internacionalización de las iniciativas emprendedoras de estos dos sectores. Asimismo, se analiza cómo las medidas de política tecnológica pueden o no ser eficientes. Para alcanzar este objetivo se hacen dos estimaciones PLS, una para cada sector para 25 países en 2007. Las estimaciones muestran que no existen grandes diferencias entre ambos sectores, excepto el en caso del capital humano que tienen un efecto mayor el caso del sector transformador.

Palabras clave:

Innovación, emprendedores, política tecnológica, determinante innovación.

\begin{abstract}
:
This paper analyzes some de factors that improve product innovation for the case of transformation sector and business services sector. Among this factors are human capital, technology, market competition, business expectations, and export intensity of entrepreneurs of these sectors. In addition, it is considered efficiency of instruments of $R \& D$ policy. To achieve these goals it is calculated two PLS estimation for 25 countries in 2007 for each sector. The estimations show that there aren't significant differences between two sector except in the case of human capital. These factors have more effects on product innovation in the case transforming sector.
\end{abstract}

Keywords:

Innovation, entrepreneurs, $R \& D$ policy, factors of innovation.

\footnotetext{
${ }^{1}$ Fac. Ciencias Económicas y Empresariales de Albacete Universidad de Castilla - La Mancha. Plaza de la Universidad, 102071 Albacete. Mariasoledad.castano@uclm.es
} 


\section{INTRODUCCIÓN}

El progreso tecnológico es el principal causante de los aumentos en la productividad de una economía y por tanto, uno de los factores esenciales que producen crecimiento económico (Galindo and Méndez 2008, Audrestch, 2005, Acs et al. 2002, Rodríguez-Pose y Crescenzi, 2006, Carrasco y Castaño, 2011). Además, el progreso tecnológico supone la introducción en el mercado de nuevos productos y la elevación de la calidad de los existentes, permiten que éstos puedan adoptarse a las nuevas exigencias de la demanda, lo que permite la supervivencia de la empresas en el mercado que son capaces de llevar a cabo proyectos innovadores. Este hecho ha llevado a que la literatura especializada se interese por determinar cuáles son las estrategias empresariales y los factores que pueden llevar a la aparición de innovaciones.

Igualmente, los decisores políticos se han preocupado por incentivarlo, debido a que el avance tecnológico supone incrementos de productividad y crecimiento económico, con los consiguientes efectos en los objetivos de política económica. Hoy en día, este tema se vuelve capital ante los procesos de globalización y deslocalización industrial.

Así, este trabajo analiza en el segundo apartado distintas posturas o modos de estudiar la innovación y qué factores son determinantes en el éxito de las actividades de I+D+i. En el tercer apartado, se examina brevemente los objetivos e instrumentos de la política tecnologica y su eficacia. Seguidamente, se realiza un análisis empírico por medio de dos estimaciones PLS para 27 países en 2007. Para finalizar con un breve apartado de conclusiones.

\section{INNOVACIÓN Y ACTIVIDAD EMPRENDEDORA}

Desde la publicación de la obra de Schumpeter (1934), los estudios que analizan los efectos de la innovación han ido creciendo debido a sus importantes efectos en crecimiento económico. Así existen estudios sobre los efectos positivos de la innovación en el crecimiento económico (Abramovitz 1986, Galindo and Méndez 2008, Audrestch 2005, Acs et al. 2002, Carrasco y Castaño, 2011, Rodríguez-Pose y Crescenzi 2006).

Asímismo, existen distintas perspectivas que intentan determinar los factores que condicionan el proceso de innovación. Según Cohen y Levin (1989) existen estudios que se centran en los inputs de la I+D, como pueden ser los gastos en I+D de las empresas, el personal empleado en actividades de I+D (Gonzalez-Pernia y Peña-Legazkue, 200, Silva, 2007, Raymond y St-Pierre, 2010) y otros que se centran en los output en el que la innovación se aproxima por sus resultados y en concreto por las patentes (Griliches,1990, Jaffe et al. 1993, Jaffe y Trajtenberg, 2002). Recientemente, la literatura aborda el estudio de los procesos de innovación sin diferenciar si es por un lado u otro (input u output), sino que se centran en la distinción entre innovación de producto y de proceso (Mairesse, 2004 y Calvo, et al. 2010).

Por su parte, el capital humano y conocimiento juegan un papel clave en la innovación (Jiménez y Sanz, 2004 y Lawson y Samson (2001). Lawson y Samson (2001) sostienen que los efectos del capital humano y el conocimiento tecnológico acumulado como recursos de la empresa que son determinantes de la innovación. Estos autores afirman que sin personas 
altamente calificadas, con actitud hacia la cooperación y capacidad innovadora, es imposible la innovación en la empresa, y sin tecnología (instalaciones modernas de manufactura, tecnologías actualizadas, tecnologías de proceso desarrolladas internamente, tecnologías de producto propias); y sin conocimiento tecnológico adecuado tampoco es posible la innovación (Zahra y Nielsen 2002, Rothaermel y Hess 2006). Sin embargo, las personas y el conocimiento tecnológico acumulado no son suficientes para determinar la innovación. En particular, el conocimiento tecnológico no es el único tipo de conocimiento necesario para la innovación. En ese sentido, Afuah (1997) y Lawson y Samson (2001) destacan entre las capacidades para la innovación el conocimiento de mercado (Silva, 2007).

Por otro lado, existen numerosos estudios que se centran en el tamaño de las empresas. Según Schumpeter (1934) la innovación y el tamaño de la empresa están relacionados positivamente. Posteriormente, Rogers (1995) confirma esta tesis, ya que sostiene que las organizaciones más grandes son más innovadoras. En cambio, Van de Ven et al. (2001) afirma que las empresas grandes tienen numerosas potencialidades para fomentar alternativas innovadoras, pero también, presentan debilidades tales como inercia organizativa y menor flexibilidad para adaptarse a los cambios ambientales, estas desventajas no se dan en las empresas pequeñas.

En línea con este argumento, se ha demostrado que numerosas innovaciones son producidas por las pequeñas empresas (Menkveld y Thurik, 1999, Acs y Audretsch 1990), ya que la innovación garantiza en gran medida la supervivencia y éxito de las organizaciones (Audretsch y Fritsch, 2003, Gonzalez-Pernia y Peña-Legazkue, 2007).

\section{ANALISIS DE LA POLÍTICA TECNOLOGICA EN LAS ACTIVIDADES DE I+D+I}

La política tecnológica puede definirse como el conjunto de medidas que adopta un gobierno con el fin de aumentar, organizar y utilizar el conocimiento científico y técnico nacional, conforme a los objetivos que el país ha asignado a su desarrollo global (Pampillón, 1980: 83). Además, el progreso tecnológico incrementa la competividad y productividad de un países incidiendo positivamente en el crecimiento econónomico y en el empleo (Roca, 2008).

La política tecnológica surge por la existencia de un fallo de mercado ya que los avances científicos y tecnológicos traen consigo una serie de externalidades, que provocan que la rentabilidad social de dichas actividades supere a la privada. Además, la información científica y técnica constituye una mercancía indivisible pues, una vez que se ha obtenido, su transmisión a terceros tiene un coste insignificante y no supone merma en la oferta disponible (Parejo, et al. 1995).

Estos rasgos hacen que el productor de nuevos conocimientos científicos y tecnológicos tenga dificultades para apropiarse de su rentabilidad a través de una simple venta en el mercado y, por tanto, esto provoca que los recursos destinados a la I+D+i sean inferiores al volumen de recursos socialmente óptimos (Griliches, 1998). En definitiva, surge un fallo de mercado (Arrow, 1962 y Nelson, 1959) que justifica la intervención del decisor político en este ámbito, por medio de los instrumentos de la política tecnológica que se exponen seguidamente. 
Entre las medidas con los que cuenta el decisor político para incentivar las actividades de I+D+i, se encuentran (Parejo, el al., 1995):

1. Medidas para incentivar la inversión en $\mathrm{I}+\mathrm{D}+\mathrm{i}$, tales como ayuda directa a la $\mathrm{I}+\mathrm{D}+\mathrm{i}$, ayudas a las universidades y centros públicos para actividades de I+D+i, subsidios directos a empresas e instituciones privadas para que realizan un esfuerzo investigador, medidas de estímulo fiscal y apoyo financiero a las empresas para el desarrollo de proyectos de innovación.

2. Medidas dirigidas a potenciar la difusión y adquisición de los conocimientos científicos y técnicos, entre otras se encuentran: la creación de infraestructuras informáticas sobre ciencia y tecnología, el fomento del capital humano, la creación de centros de investigación y/o parques científicos tecnológicos, y la participación en programas tecnológicos internacionales.

3. Fomento de un entorno favorable para la innovación de las empresas, tales como: mejorar las condiciones de competencia y acceso al mercado, protección de la propiedad industrial, regular e incentivar la transferencia de tecnología.

Así pues, la existencia de un fallo de mercado, como las medidas que se aplican para compensarlo, han provocado la aparición de numerosos estudios sobre la importancia de las actividades de $\mathrm{I}+\mathrm{D}+\mathrm{i}$. Existe una controversia entre los economístas que analizan la eficacia o deseabilidad de las medidas de apoyo a dichas actividades. Así, existen estudios que demuestran los efectos positivos de las ayudas públicas en I+D+i (Klette, et al. 2000, Trajtenberg ,2000) o incentivos fiscales (Rubio, 2010).

Por su parte, Lerner (1998), muestra que tienen efectos similares tanto las empresas subvencionadas, como las no subvencionadas en términos de empleo y ventas. Cordón y Guiterrez, (2010) llegan la conclusión que la ineficiencia de los incentivos fiscales se debe a la falta claridad de las leyes, o excesiva burocracia. Por otro lado, existen trabajos que se centran en los efectos crowding-out de sector público sobre el sector privado de las actividades de $\mathrm{I}+\mathrm{D}+\mathrm{i}$, observándose que las pymes reducen sus gastos privados en I+D+i si reciben subvenciones públicas para dichas actividades (Wallsten 2000 y Lach 2000).

\section{ANALISIS EMPÍRICO}

El objetivo de este apartado es estudiar empíricamente los determinantes de innovación de producto para el caso de los emprendedores del sector transformador y del sector servicios a empresas ${ }^{1}$, ya que existen trabajos que consideran que los emprendedores juegan un papel esencial en la innovación (Acs, et al. 1994 y Audretsch, 2005). Para ello se van a uti-

\footnotetext{
${ }^{1}$ El observatorio GEM diferencia en cuatro sector principales de actividad: extractivo, transformador, servicios a empresas y orientado al consumo. Así, el sector transformador incluye esencialmente la manufacturas y la construcción y, el sector servicios a empresas estarían actividades tales como, asesorías, agencias inmobiliarias y servicios financieros, consultoría, ...
} 
lizar datos 2 de 2007 para 25 países $^{3}$ que participan en el proyecto "Global Entrepreneurship Monitor" (GEM) (APSGLOBAL-GEM, 2007). El proyecto GEM establece como medida de la actividad emprendedora el índice TEA ${ }^{4}$ (Total Entrepreneurial Activity o índice de actividad emprendedora total).

Así, como ya se ha indicado, el objetivo de este trabajo es estudiar ciertos determinantes de la innovación de producto, para ello, se van a realizar dos estimaciones por medio de mínimos cuadrados parciales (PLS), para cada sector. La regresión PLS utiliza varias variables explicativas o proxy de un hecho económico no directamente observables, y las sintetiza y las sustituye por variables latentes (Tenenhaus, 1998).

Así, este analisis empírico intenta responder a las siguientes proposiciones analizadas en los apartados anteriores:

H1: El capital humano tiene efectos positivos en la innovación de producto y en la internacionalización de los emprendedores.

H2: La disposición de tecnologías adecuadas y recientes incentiva la innovación de producto.

H3: La competencia en los mercados y las expectativas empresariales fomentan la innovación de producto.

H4: Los emprendedores más internacionalizados innovan más.

H5: Las medidas de política económica afectan positivamente a la innovación de producto y a la internacionalización de los emprendedores.

PLS combina las características de análisis de componentes principales y regresiones múltiples, de modo que su ventaja es hacer frente a problemas de multicolinealidad (no se requiere normalidad multivariante), especialmente, esto es importante cuando se trabaja con un gran número de predictores, en comparación con el número de observaciones (es más apropiado cuando el tamaño de la muestra es pequeño ${ }^{5}$ ) (Gefen, et al. 2000; Barclay et al. 1995; Fornell, 1982, Tenenhaus, 1998).

Así, el modelo propuesto con varibles latentes es:

$$
\phi_{i 0}=\lambda_{0}+\lambda_{1} \varpi_{i 0}+\lambda_{2} \xi_{i 0}+\lambda_{3} \psi_{i 0}+\lambda_{4} \eta_{i 0}+\lambda_{5} \sigma_{i 0}+\lambda_{6} \vartheta_{i 0}+u_{i 0}
$$

donde $\phi_{i 0}$ es la innovación de producto i, $\varpi_{i 0}$ es grado de internacionalización, $\xi_{i 0}$ son las medidas de política económica, $\psi_{i 0}$ es el capital humano de los emprendedores, $\eta_{i 0}$ son

\footnotetext{
${ }^{2}$ Los datos han sido tratados con métodos de estadística descriptiva básica con el software estadístico SPSS, con el fin de obtener solamente y por separado los datos de los emprendedores del sector transformador y del sector servicios a empresas.

${ }^{3}$ Estados Unidos, Rusia, Holanda, Bélgica, Francia, España, Italia, Rumania, Suiza, Reino Unido, Noruega, Perú, Argentina, Brasil, Chile, Colombia, Islandia, Finlandia, Letonia, Serbia, Croacia, Eslovenia, Venezuela, Uruguay, Israel.

${ }^{4}$ Este indicador mide la actividad de emprendedores o iniciativas emprendedoras en un año para cualquier país que participe en este proyecto.

${ }^{5}$ Si se compara la estimación PLS con la estimación por Mínimos Cuadrados Ordinarios (MCO), para obtener los mejores estimadores insesgados el número de países que deberían integrar la muestra debería de ser mayor para una estimación MCO que mediante una estimación con PLS. Por ello, teniendo en cuenta el número de predictores considerados en este trabajo se escoge la estimación PLS para este estudio.
} 
las expectativas en el mercado nacional, $\sigma_{i 0}$ tecnologías necesarias para la actividad, $\vartheta_{i 0}$ el grado de competencia.

Para estimar esta ecuación se utiliza mínimos cuadrados parciales (PLS) con el programa SmartPLS 2.0.M3 (www.smartpls.de) y con el método estructural o regresión múltiple (Path Weihting) (Tenenhaus, 1998).

A continuación se explican los indicadores que forman las variables latentes utilizadas en el modelo:

Para medir la variable latente innovación de producto $\phi_{i 0}$ se utiliza el ítem de la encuesta GEM, “¿cuántos consumidores consideran su producto nuevo?” escogiendo las opciones "todos" y "algunos", y diferenciado en cada modelo por el sector correspondiente.

Por su parte, la variable latente grado de internacionalización $\varpi_{i 0}$ se mide a través del ítem de la encuesta GEM "intensidad exportadora”, opción 25\%-75\% y la pregunta "¿al menos el $25 \%$ de los clientes de la empresa son extranjeros?".

$\psi_{i 0}$ mide el capital humano de los emprendedores por medio de la variable conocimiento, habilidades y experiencia para emprender, y así como el indicador cuantos emprendedores del sector tienen estudios universitarios.

La variable latente competencia $\eta_{i 0}$ incluye las expectativas de los emprendedores en su mercado nacional y el motivo que llevó a emprender, "TEA por oportunidad".

La variable tecnología, recoge indicadores de tecnologías requeridas y disponibles muy recientes y $\vartheta_{i 0}$ mide el grado de competencia por medio del ítem de la encuesta GEM ¿cuántos competidores ofrecen mismo producto?, respuesta "pocos", el ítem expansión de mercado la opción: "alguna con nuevas tecnologías". Todas las variables se diferencian según el sector al que pertenecen.

Por último, variable latente $\xi_{i 0}$, medidas de política económica está compuesta por cuatro preguntas ${ }^{7}$ de la encuesta a expertos del observatorio GEM $^{8}$ (NES-GEM, 2007). En este caso, los datos son los mismos para los dos modelos, no se diferencia por sectores ya que son encuestas a expertos.

Las figuras 1 y 2 recogen el Path diagrama para cada uno de los sectores, donde se observan los principales efectos y relaciones entre las variables.

\footnotetext{
${ }^{6}$ El observatorio GEM diferencia, entre TEA por oportunidad, TEA por necesidad y TEA por otros motivos (Bosma, et al. 2008).

${ }^{7}$ Estas preguntas son: si existen suficientes ayudas públicas para incentivar la actividad emprendedora; las políticas públicas ayudan a las nuevas empresas; los parques tecnológicos y las incubadoras de empresas ayudan a las nuevas empresas; la eficiencia de las universidades y centro de investigación públicas en la transferencia de tecnologías hacia las iniciativas emprendedoras.

${ }^{8}$ En este caso la variable es una media de las respuestas de estos expertos, donde el valor mínimo es 1 y el máximo 5.
} 
Figura 1

Determinantes de la Innovación De Producto para Los Emprendedores Del Sector Transformador

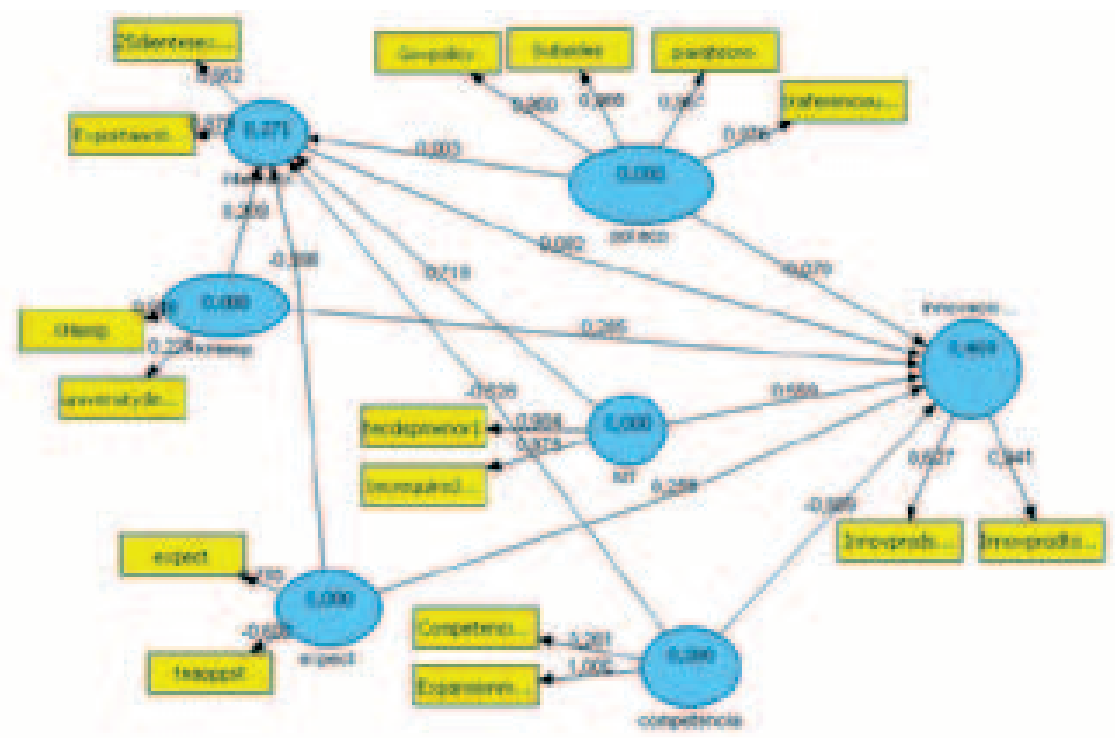

Figura 2

Determinantes de la innovación de producto para los emprendedores del sector servicios a empresas

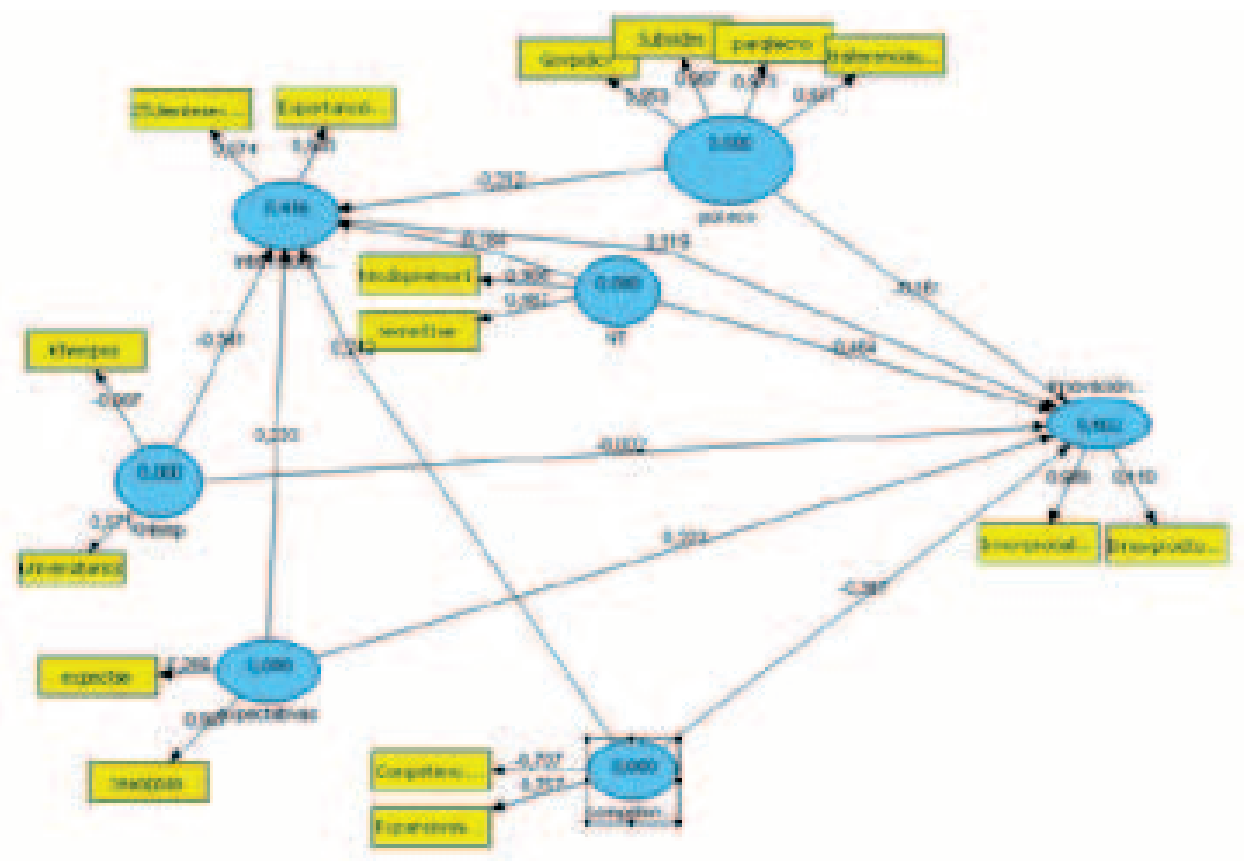


La Tabla 1 recoge las medidas de validez de los modelos: la varianza extraída media (AVE) y el $\mathrm{R}^{2}$. Según Fornell y Larcker, 1981, existe validez convergente cuando AVE9> 0,5 . Todas las variables cumplen este criterio excepto la variable latente expectativas, y la variable latente capital humano en el caso de modelo del sector servicios a empresas.

Tabla 1

Varianza extraída media (AVE) de los constructos de los modelos

\begin{tabular}{lcccc}
\hline & $\begin{array}{c}\text { AVE. S. } \\
\text { Transformador }\end{array}$ & $\begin{array}{c}\text { AVE. S. Servicios } \\
\text { a empresas }\end{array}$ & $\begin{array}{c}\mathrm{R}^{2} \mathrm{~S} . \\
\text { Transformador }\end{array}$ & $\begin{array}{c}\mathrm{R}^{2} \text { S. Servicios a } \\
\text { empresas }\end{array}$ \\
\hline KHemp & 0,572472 & 0,473991 & 0,000 & 0,000 \\
NT & 0,959636 & 0,973643 & 0,000 & 0,000 \\
competencia & 0,533844 & 0,536601 & 0,000 & 0,000 \\
expect & 0,488866 & 0,446805 & 0,000 & 0,000 \\
innovacionproducto & 0,639712 & 0,593930 & 0,469234 & 0,602249 \\
internacionalización & 0,839531 & 0,935109 & 0,270775 & 0,416126 \\
pol.eco & 0,935468 & 0,935889 & 0,000 & 0,000 \\
\hline
\end{tabular}

La tabla 2 y 3 muestran las matrices de correlaciones de los dos modelos propuestos.

Tabla 2

Matrix de correlaciones entre las variables latentes: sector transformador

\begin{tabular}{|c|c|c|c|c|c|c|c|}
\hline & KHemp & NT & $\begin{array}{l}\text { competen- } \\
\text { cia }\end{array}$ & expect & $\begin{array}{l}\text { Innovación } \\
\text { producto }\end{array}$ & $\begin{array}{l}\text { internacio- } \\
\text { nalización }\end{array}$ & pol.eco \\
\hline KHemp & 1,000000 & & & & & & \\
\hline NT & $-0,172097$ & 1,000000 & & & & & \\
\hline competencia & $-0,212507$ & 0,941066 & 1,000000 & & & & \\
\hline expect & 0,299283 & $-0,461788$ & $-0,515000$ & 1,000000 & & & \\
\hline innovacionproducto & 0,423873 & $-0,398341$ & $-0,516531$ & 0,499890 & 1,000000 & & \\
\hline internacionalización & 0,082848 & 0,365856 & 0,304779 & $-0,387382$ & $-0,060019$ & 1,000000 & \\
\hline pol.eco & 0,531883 & $-0,223398$ & $-0,184272$ & 0,122440 & 0,152065 & 0,003569 & 1,000000 \\
\hline
\end{tabular}

\footnotetext{
9 Se define por Fornell y Larcker, (1981) como $\begin{array}{r}\operatorname{AVE}_{i}>\rho_{i j}^{2} \\ \operatorname{AVE}_{j}>\rho_{i j}^{2}\end{array}$
} 
Tabla 3

Matrix de correlaciones entre las variables latentes: sector servicios a empresas

\begin{tabular}{|c|c|c|c|c|c|c|c|}
\hline & KHemp & NT & $\begin{array}{l}\text { compe- } \\
\text { tencia }\end{array}$ & expect & $\begin{array}{c}\text { Inno- } \\
\text { vación } \\
\text { servemp }\end{array}$ & $\begin{array}{l}\text { interna- } \\
\text { cionaliza- } \\
\text { ción }\end{array}$ & pol.eco \\
\hline KHemp & 1,000000 & & & & & & \\
\hline NT & 0,125264 & 1,000000 & & & & & \\
\hline competencia & 0,034881 & 0,211573 & 1,000000 & & & & \\
\hline expectativas & $-0,502205$ & $-0,571535$ & 0,017130 & 1,000000 & & & \\
\hline innovaciónservemp & $-0,261864$ & $-0,644650$ & $-0,427927$ & 0,500110 & 1,000000 & & \\
\hline internacionalización & $-0,467795$ & 0,086659 & 0,275271 & 0,274533 & 0,096778 & 1,000000 & \\
\hline pol.eco & 0,132681 & $-0,087321$ & $-0,007063$ & 0,092445 & $-0,140131$ & $-0,353122$ & 1,000000 \\
\hline
\end{tabular}

Tablas 4 y 5 muestra las cargas cruzadas de los modelos analizados, esta tabla recoge la asignación de los indicadores a los constructos.

Tabla 4

Tabla de cargas cruzadas (cross-loading): sector transformador

\begin{tabular}{lccccccc}
\hline & KHemp & NT & $\begin{array}{c}\text { competen- } \\
\text { cia }\end{array}$ & expect & $\begin{array}{c}\text { Innovación } \\
\text { producto }\end{array}$ & $\begin{array}{c}\text { internacio- } \\
\text { nalización }\end{array}$ & pol.eco \\
\hline 25clientesextranjeros & $-0,010638$ & 0,450018 & 0,411625 & $-0,462681$ & $-0,166128$ & 0,951994 & $-0,016765$ \\
\hline CompetenciaPoca & 0,031404 & 0,296750 & 0,260953 & 0,108784 & $-0,050025$ & $-0,109328$ & 0,220716 \\
\hline $\begin{array}{l}\text { Expansionmerca- } \\
\text { doalgunaNT }\end{array}$ & $-0,210646$ & 0,941945 & 0,999796 & $-0,509807$ & $-0,514652$ & 0,300765 & $-0,178610$ \\
\hline Exportanción2575 & 0,212212 & 0,163208 & 0,078758 & $-0,194325$ & 0,116959 & 0,879074 & 0,034534 \\
Govpolicy & 0,465520 & $-0,246782$ & $-0,188416$ & 0,190710 & 0,176092 & $-0,072847$ & 0,959629 \\
\hline Innovprodsome & 0,150862 & $-0,310397$ & $-0,331982$ & 0,269646 & 0,627392 & $-0,316171$ & $-0,006795$ \\
\hline Innovprodtodos & 0,448875 & $-0,348660$ & $-0,482710$ & 0,489568 & 0,941172 & 0,064377 & 0,187470 \\
\hline KHemp & 0,945958 & $-0,217594$ & $-0,249378$ & 0,408271 & 0,399174 & 0,026222 & 0,535693 \\
\hline Subsides & 0,576193 & $-0,123769$ & $-0,096046$ & 0,033298 & 0,139680 & 0,135144 & 0,965856 \\
\hline expect & 0,369955 & $-0,277553$ & $-0,309010$ & 0,784500 & 0,316481 & $-0,397908$ & 0,027701 \\
\hline parqtecno & 0,540258 & $-0,243922$ & $-0,207329$ & 0,065986 & 0,129297 & 0,009980 & 0,967126 \\
\hline teaoppst & $-0,006079$ & 0,387050 & 0,432327 & $-0,601906$ & $-0,398347$ & 0,112111 & $-0,161719$ \\
\hline tecdispmenor1 & $-0,197460$ & 0,983951 & 0,974046 & $-0,503533$ & $-0,454999$ & 0,362122 & $-0,244287$ \\
\hline tecrequiredmenor1 & $-0,132988$ & 0,975251 & 0,858195 & $-0,389449$ & $-0,310356$ & 0,354152 & $-0,187543$ \\
traferenciaunivemp & 0,485712 & $-0,247277$ & $-0,224377$ & 0,165885 & 0,133894 & $-0,044372$ & 0,976102 \\
\hline universitydegree & 0,223847 & 0,126168 & 0,097760 & $-0,309171$ & 0,100269 & 0,175273 & 0,021222 \\
\hline & & & & & & & \\
\hline
\end{tabular}


Tabla 5

Tabla de cargas cruzadas (cross-loading): sector servicios a empresas

\begin{tabular}{|c|c|c|c|c|c|c|c|}
\hline & KHemp & NT & $\begin{array}{l}\text { competen- } \\
\text { cia }\end{array}$ & Expect. & $\begin{array}{c}\text { Innovación } \\
\text { servemp }\end{array}$ & $\begin{array}{l}\text { internacio- } \\
\text { nalización }\end{array}$ & pol.eco \\
\hline 25 clientesextranjeros & $-0,453334$ & 0,069217 & 0,211150 & 0,302371 & 0,176367 & 0,974261 & $-0,467029$ \\
\hline CompetenciaPoca & $-0,263442$ & 0,076036 & $-0,707056$ & 0,043778 & 0,308822 & $-0,181038$ & $-0,006320$ \\
\hline $\begin{array}{l}\text { Expansionmerca- } \\
\text { doalgunaNT }\end{array}$ & $-0,194164$ & 0,368619 & 0,757148 & 0,064599 & $-0,318227$ & 0,220978 & $-0,015799$ \\
\hline Exportancións 2575 & $-0,451963$ & 0,102129 & 0,335279 & 0,219959 & $-0,009442$ & 0,959705 & $-0,185568$ \\
\hline Govpolicy & 0,127852 & $-0,123264$ & $-0,091262$ & 0,029305 & $-0,065175$ & $-0,367468$ & 0,952683 \\
\hline Innovprodalgunos & $-0,261640$ & $-0,628722$ & $-0,465353$ & 0,471448 & 0,987838 & 0,057616 & $-0,098214$ \\
\hline Innovprodtodos & $-0,009800$ & $-0,122424$ & 0,225574 & 0,199211 & 0,109704 & 0,253440 & $-0,272440$ \\
\hline Khempse & $-0,066524$ & $-0,014469$ & 0,413963 & 0,061492 & $-0,048194$ & 0,163642 & $-0,201006$ \\
\hline Subsides & 0,132624 & $-0,114726$ & 0,076002 & 0,156881 & $-0,171216$ & $-0,284873$ & 0,966932 \\
\hline expectse & 0,248709 & $-0,162076$ & 0,362061 & $-0,266225$ & $-0,114845$ & $-0,235825$ & $-0,101693$ \\
\hline parqtecno & 0,141857 & $-0,031742$ & 0,036751 & 0,118195 & $-0,215965$ & $-0,354691$ & 0,973034 \\
\hline teaoppse & $-0,405288$ & $-0,655664$ & 0,175676 & 0,907047 & 0,461615 & 0,177931 & 0,050183 \\
\hline tecdispmenor 1 & 0,120641 & 0,986046 & 0,168494 & $-0,580389$ & $-0,623761$ & 0,050179 & $-0,047990$ \\
\hline tecred 1 se & 0,126418 & 0,987421 & 0,247022 & $-0,548362$ & $-0,647836$ & 0,119069 & $-0,122421$ \\
\hline traferenciaunivemp & 0,109583 & $-0,077481$ & $-0,046316$ & 0,056206 & $-0,082925$ & $-0,352365$ & 0,976831 \\
\hline universitarios & 0,971368 & 0,120216 & 0,133002 & $-0,481139$ & $-0,269989$ & $-0,422847$ & 0,083123 \\
\hline
\end{tabular}

Finalmente, la tabla 6 recoge los efectos directos entre variables latentes en los modelos propuestos.

Tabla 6

Efectos directos entre variables latentes diferenciado por sectores

\begin{tabular}{lcccc}
\hline & \multicolumn{2}{c}{ Sector transformador } & \multicolumn{2}{c}{ Sector servicios a empresas } \\
\hline & $\begin{array}{c}\text { Innovación } \\
\text { producto }\end{array}$ & internacionalización & $\begin{array}{c}\text { Innovación } \\
\text { servemp }\end{array}$ & internacionalización \\
KHemp & 0,302474 & 0,209443 & $-0,002112$ & $-0,340732$ \\
NT & 0,618175 & 0,719319 & 0,463939 & 0,184361 \\
competencia & $-0,931737$ & $-0,527982$ & $-0,367417$ & 0,241950 \\
expect & 0,223529 & $-0,390214$ & 0,222433 & 0,233454 \\
innovacionproducto & & & & $-0,311688$ \\
internacionalización & 0,081694 & & 0,119046 & $-0,161482$ \\
\hline pol.eco & $-0,069779$ & 0,003349 & & \\
\hline
\end{tabular}


Así, se observa que el capital humano tiene efectos positivos sobre la innovación de producto (Hadjimanolis, 2000), y la internacionalización (Carrasco y Castaño, 2011) en el modelo para el sector transformador. Pero tiene efectos negativos en el caso del sector servicios a empresas, pero estos resultados no son significativos ya que el AVE del modelo para esta variable latente es menor de 0.5. Así pues, la hipótesis H1, se confirmaría para el caso del sector transformador, donde el capital humano de los emprendedores juega un papel esencial, pero esto no ocurre en el caso del sector servicios a empresas ya que la variable no es significativa, esto hace que no se puedan sacar resultados concluyentes respecto al signo de esta variable e indicaría que el capital humano de los emprendedores en este sector no es una variable relevante.

La variable tecnología tiene un efecto positivo y significativo en los dos modelos propuestos, esto confirma la hipótesis $\mathrm{H} 2$ y las relaciones antes analizadas, que sostienen que son necesarias tecnologías adecuadas y nuevas para facilitar la innovación (Silva, 2007). Sin embargo, su efecto es mucho mayor en el caso del sector transformador, esto podría deberse a que es necesario para que la tecnología tenga sus efectos plenos en la innovación es necesario un capital humano adecuado para aprovecharla, y en el sector servicios debe existir alguna deficiencia en el capital humano por los resultados obtenidos en el segundo modelo.

La competencia tiene un efecto negativo en los dos sectores, esto es debido a los indicadores que forman la variable latente, es decir, se mide el caso en que los emprendedores tienen "poca competencia" y, por tanto, si los emprendedores que no sienten la presión de sus competidores entonces tienden a innovar menos, este resultado confirma la hipótesis H3. La variable expectativa en los mercados nacionales tienen efecto positivo en los dos sectores, pero los AVE en este casos son algo menores de 0.5 .

Según Kafouros et al. (2008) las empresas más internacionalizadas son las que más innovan, estos modelos confirmarían la hipótesis $\mathrm{H} 4$, en ambos casos, el efecto sería positivo e incluso superior en el caso de los emprendedores de sector servicios. Además, este resultado sería muy positivo ya que indicaría que sector servicios está haciendo una apuesta por abrirse a los mercados internacionales y esto les hace ser más innovadores para competir en los mercados internacionales.

Por último, la variable latente medidas de política económica tiene un efecto negativo en la innovación de producto en ambos casos. Estos efectos se explican por las variables escogidas como indicadores, estas preguntas a expertos recogen la opinión de estos sobre el adecuado diseño de las medidas de política tecnológica para incentivar la innovación. En casi todos los ítems escogidos, los expertos consideran que las políticas tecnológicas están mal diseñadas o son insuficientes, esto explica los signos negativos. Por tanto, si estas medidas estuvieran mejor diseñadas serían esperables unos resultados positivos acorde a las tesis antes analizadas (Klette, et al. 2000, Trajtenberg, 2000). Asimismo, se observan que los efectos sobre la internacionalización son negativos o casi nulos en ambos sectores. Esto se puede explicar igualmente por el mal diseño de estas medidas de política tecnológica. De tal forma que la hipótesis H5, no puede ser confirmada, ya que los modelos indican que las medidas política económica mal diseñadas tienen efectos negativos, pero se desconocen los efectos si estuvieran bien diseñadas. 


\section{CONCLUSIONES}

Este trabajo ha realizado una breve revisión de la literatura económica sobre distintos estudios que abordan el complejo y esencial proceso de la innovación tecnológica para alcanzar los objetivos de política económica, en especial, el crecimiento económico.

Además, se analizan con qué medidas de política tecnológica cuenta el decisor político para incentivar las actividades de I+D+i, así como la controversia académica respecto a su eficacia.

Por su parte, el análisis empírico obtiene los siguientes resultados: no se observan grandes diferencias entre los dos sectores excepto en el caso de la variable capital humano. El efecto de ésta es mayor en caso del sector transformador, y además, en el caso sector servicios a empresas esta variable latente no es significativa.

Por su parte, la disposición de nuevas tecnologías tiene efectos positivos en ambos sectores, pero es mucho mayor para el sector transformador que para el sector servicios a empresas, esto puede ser debido a que los requerimiento de tecnologías sean mayores en el primer sector que en el segundo y, además, por la combinación adecuada que se da en primer modelo entre capital humano y tecnología.

Por otro lado se observa que las empresas más expuestas a la competencia y las que se dirigen más a los mercados internacionales son también las que más innovadoras, ya que la innovación es esencial para sobrevivir en mercados muy competitivos.

Por último, se puede apreciar que las medidas de política tecnológica deben diseñarse mejor, puesto que las políticas económicas inadecuadas tienen efectos negativos sobre la innovación de producto. Así pues, es necesario hacer un esfuerzo en un diseño adecuado de éstas para ajustarse a las necesidades de los emprendedores de ambos sectores y conseguir los objetivos marcados, tales como, incremento de las actividades de I+D+i, la transferencia de tecnología de la universidad a las empresas,..., para así conseguir aumentar la productividad y la competitividad de estas iniciativas emprendedoras.

\section{REFERENCIAS BIBLIOGRÁFICAS}

ABRAMOVITZ, M. (1986): "Catching-up, forging ahead and falling be-hind", Journal of Econo-

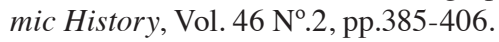

ACS, Z.J.; ANSELIN, L. y VARGA, A. (2002): "Patents and innovations count as measures of regional production of new knowledge" Research Policy, 31: 1069-4085.

ACS, Z.J. y AUDRETSCH, D. (1990): Innovation and Small Firms, Cambridge: MIT Press.

ACS, Z.J.; AUDRETSCH, D. y FELDMAN, M. (1994): "R\&D spillovers and recipient firm size", The Review of Economics and Statistics, 76, 336-340.

AFUAH, A. (1997): La Dinámica de la Innovación Organizacional. México: Oxford University Press.

ARROW, K.J. (1962): "Economic Welfare and the Allocation of Resources for Invention", in NELSON, R.R. (ed.): The Rate and Direction of Inventive Activity, Princeton University Press, Princeton, 1962, pp.609-625.

AUDRETSCH, D. (2005): "The Emergence of Entrepreneurial Economics" en VINIG, R.C.W. VAN DER VOORT (eds): Research on Technological Innovation, Management and Policy, Vol. 9 , pp. 37-54. 
AUDRETSCH, D. y FRITSCH, M. (2003): "Linking Entrepreneurship to Growth: The Case of West of Germany", Industry and Innovation, Vol. $10 \mathrm{~N}^{\circ} 1$, pp. 65-73.

BARCLAY, D.; HIGGINS, C. y THOMPSON, R. (1995): “The Partial Least Squares (PLS) Approach to Causal Modeling. Personal Computer Adoption and Use as an Illustration", Technology Studies, Special Issue on Research Methodology, Vol. 2 N², pp. 285-309.

BOSMA, N.; ACS, Z.J.; AUTIO, E.; CODURAS, A. y LEVIE, J. (2008): Global Entrepreneurship Monitor: 2008 Executive Report, London: London Business School.

CALVO, J.J. (2010) (coord.): Estudios sobre la innovación tecnológica en España, Uned: Madrid.

CARRASCO, I. y CASTAÑO, M.S. (2011): Innovación e internacionalización de los emprendedores en España, Economía Industrial, Vol. 380, pp. 79-86.

COHEN, M. y LEVIN R.C. (1989): "Empirical studies of innovation and market structure", En R. C. Schmalensee and R. Willig (eds.), Handbook of Industrial Organization, Amsterdam: Elsevier, pp. 1059-1107.

CORDÓN, T. y GUTIERREZ, M. (2010): "Incentivos fiscales a la investigación, el desarrollo y la innovación técnologica", Clm-economía, 16, pp. 281-310.

FORNELL, C. (1982): "A second generation of multivariate analysis: An Overview". In FORNEL, C. (ed.): A Second Generation of Multivariate Analysis, New York: Praeger Publishers.

FORNELL, C. y LARCKER, D. (1981): "Evaluating structural equations models with unobservable variables and measurement error", Journal of Marketing Research, Vol.18, pp. 39-50.

GALINDO, M.A. y MÉNDEZ, M.T. (2008): Emprendedores y objetivos de política económica, Información Comercial Española, 841, pp. 29-40.

GEFEN, D.; STRAUB, D.W. y BORDEAU, M.C. (2000): "Structural Equation Modelling and Regression: Guidelines for Research Practise", Communications of the Association of Information Systems, Vol.4, No.7, pp.1-79.

GEM (2007): GEM: Dataset. http://www.gemconsortium.org/

GONZÁLEZ-PERNÍA, J.L. y PEÑA-LEGAZKUE, I. (2007): "Determinantes de la capacidad de innovación de los negocios emprendedores en España”, Economía Industrial, Vol. 363, pp.129147.

GRILICHES, Z. (1990): "Patent statistics as economic indicators: a survey" Journal of Economic Literature Vol. 28, No. 4, pp. 92-116.

GRILICHES, Z. (1998): $R \& D$ and Productivity, the Econometric Evidence. University of Chicago Press: Chicago.

HADJIMANOLIS, A. (2000): "A resource-based view of innovativeness in small firms" Technology Analysis \& Strategic Management, Vol.12, №. 2, pp.263-281.

JAFFE, A.B. y TRAJTENBERG, M. (2002): Patents, citations, and innovations: a window on the knowledge economy, MIT Press: Cambridge.

JAFFE, A.B.; TRAJTENBERG, M. y HENDERSON, R. (1993): "Geographic localization of knowledge spillovers as evidenced by patent citations", Quarterly Journal of Economics Vol. 63, pp. 577-598.

JIMÉNEZ, J.D. y SANZ, V.R. (2004). "Determinantes del éxito de la innovación", Revista de Empresa, Vol. 7, pp. 24-38.

KAFOUROS, M.I.; BUCKLEY, P.J.; SHARP, J.A. y WANG, C. (2008): "The role of internationalization in explaining innovation performance", Technovation Vol. $28 \mathrm{~N}^{\circ}$ (1-2), pp. 63-74.

KLETTE, T.J.; MØEN, J. y GRILICHES, Z. (2000): "Do subsidies to commercial R\&D reduce market failures? Microeconometric evaluation studies, Research Policy, Vol. 29, pp. 471-495.

LACH, S. (2000): "Do R\&D Subsides Stimulate or Displace Private R\&D? Evidence from Israel", NBER Working Paper Series, n. 7943, http:/www.nber.org/papers7w7943.

LAWSON, B. y SAMSON, D. (2001): "Developing innovation capability in organizations: a dynamic capabilities approach”. International Journal of Innovation Management, Vol. 5 No. 3, pp. 377-400. 
LERNER, J. (1998): "The government as venture capitalist: the long-run impact of the SBIR program". Mimeo. Harvard University.

MAIRESSE, J. (2004): Knowledge Management, Innovation, and Productivity: A Firm Level Exploration Based on French Manufacturing" NBER Working Paper $\mathrm{N}^{\circ} .10237$.

MENKVELD, B. y THURIK, R. (1999): “Firm Size and Efficiency in Innovation: Reply”, Small Business Economics, Vol. 12 N $^{\circ}$. 1, pp. 97-101.

NELSON, R. (1959): “The Simple Economics of basic Scientific Research", Journal of Political Economy, pp. 297-306.

PAMPILLÓN, R. (1980): "Hacia una sistematización de la política tecnológica", Revista de Economía Política, Vol. 84, pp. 87-116.

PAREJO GAMIR, J.A.; CALVO, A. y PAUL, J. (1995): La Política Económica de reformas estructurales, Centro de estudios Ramón Areces: Madrid

RAYMOND, L. y ST-PIERRE, J. (2010): "R\&D as a determinant of innovation in manufacturing SMEs: An attempt at empirical clarification", Technovation, Vol. 30, $\mathrm{N}^{\circ}$ 1, pp. 48-56

ROCA, A. (2008): "Las políticas de competitividad: industria, empresa y tecnología”, en JORDÁN, J.M: Economía de la Unión Europea, Editorial Thonson-Civitas: Navarra

RODRÍGUEZ-POSE, A. y CRESCENZI, R. (2006): "R\&D, innovation systems and the genesis of regional growth in Europe". Università degli Studi Roma Tre, Dipartimento di Economia, Working Paper $\mathrm{N}^{\circ} 67$.

ROGERS, E. (1995): Diffusion of Innovations, New York: The Free Press.

ROTHAERMEL, F.T. y HESS, A.M. (2006): "Building dynamic capabilities: Innovation driven by individual, firm, and network level effects". Working Paper, Georgia Institute of Technology, Atlanta, GA, USA.

RUBIO, J.J. (2010): "Incentivos fiscales a la inversión empresarial en el impuesto de sociedades", Clm-economía, Vol. 16, pp. 257-280.

SCHUMPETER, J. (1934): The theory of economic development: An inquiry into profits, capital credit, interest and the business cycle, Cambridge, MA, US: Harvard University Press.

SILVA, A. (2007): "Determinantes de la innovación en la empresa" ANALES de la Universidad Metropolitana, Vol. $7 \mathrm{~N}^{\circ} .2$, pp. 53-71.

TENENHAUS, M. (1998): La régression PLS: théorie et pratique, Technip: París

TRAJTENBERG, M. (2000): "R\&D Policy in Israel: An Overview and Reassessment”, The Foeder, Institute for Economic Research, DP 7.

VAN DE VEN, A.H.; POLLEY, D.E.; GARUD, R. y VENKATARAMAN, S. (2001): El Viaje de la Innovación. México: Oxford University Press.

WALLSTEN, S. J. (2000): “The Effects of Government-Industry R\&D Programs on Private R\&D: The Case of the Small Business Innovation Research Program", RAND Journal of Economics, pp. 82-100.

ZAHRA, S.A. y NIELSEN, A. (2002). "Sources of capabilities, integration and technology commercialization”, Strategic Management Journal, Vol. 23, pp. 377-389. 